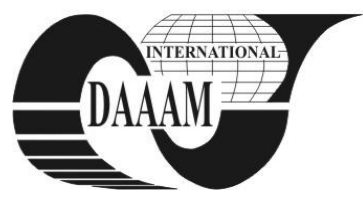

Annals of DAAAM for 2011 \& Proceedings of the 22nd International DAAAM Symposium, Volume 22, No. 1, ISSN 1726-9679 ISBN 978-3-901509-83-4, Editor B. Katalinic, Published by DAAAM International, Vienna, Austria, EU, 2011 Make Harmony between Technology and Nature, and Your Mind will Fly Free as a Bird Annals \& Proceedings of DAAAM International 2011

\title{
SIMULTANEOUS LOCALIZATION AND MAPPING FOR A WHEELCHAIR USING ELECTROMIOGRAPHIC SIGNALS
}

\author{
LUPEA, D[iana]; MAN, S[ergiu] I[onut] \& LAZEA, G[heorghe]
}

\begin{abstract}
The need of human helping robots is imposed by the nowadays trend towards protecting the physically impaired people. New researches in this area have arisen in the past years. In the next few lines an overview of the state of the art in this domain will be made. The main objective in this paper is to demonstrate the simultaneous localization for a wheelchair, using elecromiographic signals. So far the research on robots used for helping disabled people has been focusing mainly on replacing limbs by robotic prosthesis or surgery

Key words: electromiographic signals, autonomous vehicle, simultaneous localization and mapping, human-robot cooperation
\end{abstract}

\section{INTRODUCTION}

The service robotics area is predicted to be a growing market in the future years. Therefore, many research projects are concerned with developing robots to be used in performing tasks for humans. These types of robots have to be able to complete complex tasks like localization and path planning in dynamic, complex environments, or even a natural human robot interaction.

Electronic controlled wheelchairs offer the possibility of covering long distances for disabled people with spinal cord injuries or other neuromuscular problems with little physical effort. However, if the hand or arm of the patient is affected, the joystick used to control the wheelchair becomes useless. A solution is provided by the use of electromiographic (EMG) signals. The EMG signal represents the electrical manifestation associated with muscle contraction. The EMG signal can be collected from skin surface proving to be an efficient for the control of arm and hand prosthesis because EMG signals can be collected from the remaining muscles in the limb stump (Tsui et al., 2007; Jeung-Su et al., 2003).

There are still many aspects to be solved regarding this research area. Aspects such as safety requirements considering these autonomous robots which have to work in environments with humans, or safe human robot interraction. This paper proposes a system that takes into consideration these aspects, and proposes a improved solution. The main ideea is to have a autnomous wheelchair which is controlled for navigation purposes by EMG signals taken from the forearm of the user, while performing tasks such as Simultaneous Localization and Mapping (SLAM) Fig. 1 a, such that the obtained maps could be used for an easier navigation pourposes.

\section{LITERATURE OVERVIEW}

The number of robots in our lives has increased greatly in the past years. Robots which are helping humans vary from surgical robots to robots designed to clean our houses. Describing a few prototype examples would demonstrate the utility of the research in this field. One area of research is represented by robots which interract daily with humans, one example being delivery robots. An example was proposed in
(Wang et al., 2009), being designed with the concept of being able to take transport tasks for some complex medical environment in the future. Another example is the TO robot, a "human symbiotic robot" designed assist with nursing care and housekeeping. A concept closer to the one that is going to be presented in this paper is the one from (Cheein et al., 2010), who describes a wheelchair system performing Extended Kalman Filter SLAM, or the one from (Sembera et al., 2009).

In turn our solution is represented by a system which controls the wheelchair directly, for navigation tasks, based on the recorded EMG signals, without intermediate devices. At the same time the system is performing particle filter based SLAM, the maps obatined this way being usable atferwards for an aoutnomous navigation if the user wants.

\section{SYSTEM OVERVIEW}

\subsection{EMG signal extracting protocol}

It was considered that the subject should be able to associate intuitive hand movements to control the direction of the wheelchair so the movements of each finger was associated with a direction: left - index finger, right - ring finger, forward - middle finger, stop - little finger, rest - same as previous.

The EMG signals were collected from the right forearm of a healthy subject. Four EMG sensors were placed in positions were the EMG signals was found to be particular high for a finger flexion as illustrated in Fig. 1 b. A Bagnoli 4 (Delsys Inc.) device in combination with a NI6009 DAQ was used to acquire the EMG signals (gain $=1000$, sampling frequency $=$ 1000). The subject was placed in front of a computer with the application running and 20s of EMG signals were collected for each finger contraction resulting in 20x1000x5 data points.

\subsection{The classification algorithm}

The acquired EMG signal was divided into data blocks of $250 \mathrm{~ms}$, for each block the root mean square of the signal being computed as in (1). The resulting data was used to train a neural network classifier into five movement classes - corresponding to each finger contraction and to the rest state.

$$
R M S=\sqrt{1 / N \sum_{i=1}^{N} s\left(x_{i}\right)^{2}}
$$

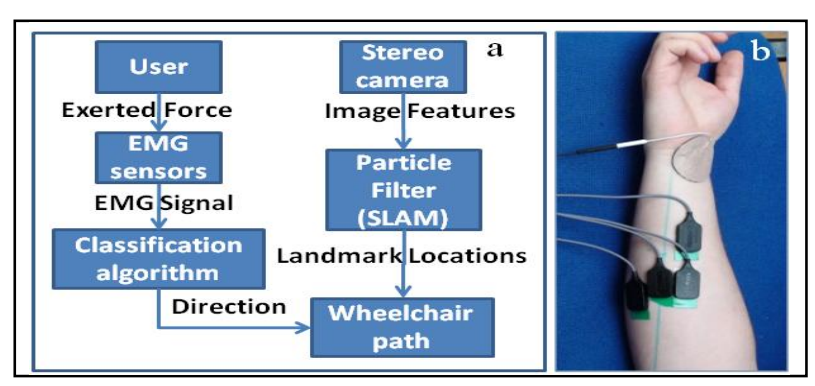

Fig. 1. (a) The structure of the system; (b) The placement of the electrodes on the subject's forearm 
The application (Fig. 2 a) contains three sections: the result of the classification algorithm indicating a direction (upper left corner), the raw EMG signals collected from the user (upper right corner) and the representation of the EMG signal amplitude as recorded by the electrodes. The subject was then asked to match the indication provided by the application (Fig.2 a) and to replicate the movement exerted during the data acquisition process.

\subsection{The Vision System}

The task of this system is to detect the image features, using a stereo camera, on both of the image pair synchronously. Our algorithm uses image features detector and descriptor called SURF. SURF is a method, known in the field of computer vision to detect interest points on images. The algorithm is based on the Fast-Hessian detector, which computes the determinant of the Hessian matrix, equation 2:

$$
\mathrm{H}(p, \sigma)=\left[\begin{array}{ll}
\mathrm{L}_{\mathrm{xx}}(p, \sigma) & \mathrm{L}_{\mathrm{xy}}(p, \sigma) \\
\mathrm{L}_{\mathrm{xy}}(p, \sigma) & \mathrm{L}_{\mathrm{yy}}(p, \sigma)
\end{array}\right]
$$

Where with $\mathrm{L}_{\mathrm{xx}}(p, \sigma)$ is denoted the convolution of the second order Gaussian derivative $\partial^{2} g(\sigma) / \partial x^{2}$ of the image at point $p(x, y)$ and scale $\sigma$ in the $x$ direction. $\mathrm{L}_{x y}(p, \sigma)$ and $\mathrm{L}_{y y}(p, \sigma)$ are calculated similarly. The reason we chose the SURF algorithm is because it performs fast beacuse approximates the Laplacian of Gaussian with a box filter representation. The box filter allows a performance increase in time when they are computed on integral images.

\subsection{The Particle Filter SLAM}

Particle filters are mathematical models that represent the probability distribution as a set of discrete particles which occupy the state space. In the update step a new particle distribution given motion model and controls applied is generated. The used algorithm, FastSLAM maintains a set of particles. In order to create a particle set at the current time, we sample from the motion model particles into a temporary set. Then compute the importance factor weights, and then based on a threshold decide which particles enter the final set. We obtain the estimation of the landmark locations. The posterior update depends on whether the k-th landmark has been observed or not. FastSLAM implements the update equation (3) by means of an EKF.

$$
p\left(\theta_{k} \mid s_{t}, z_{t}, n_{t}\right)\left\{\begin{array}{c}
p\left(z_{t} \mid \theta_{t}, s_{t}, n_{t}\right) p\left(\theta_{k} \mid s_{t-1}, z_{t-1}, n_{t-1}\right), k=n_{t} \\
p\left(\theta_{k} \mid s_{t-1}, z_{t-1}, n_{t-1}\right) \text { otherwise }
\end{array}\right.
$$

\section{EXPERIMENTAL RESULTS, CONCLUSIONS AND FUTURE WORK}

Our proposal was to create a system which uses EMG signals taken from a human in order to navigate through an environment, while performing SLAM. First the results for the classification algorithm will be presented and then the ones for the FastSLAM algorithm.

For the EMG signal extracting system a user command was considered valid when consecutive outputs of the classification

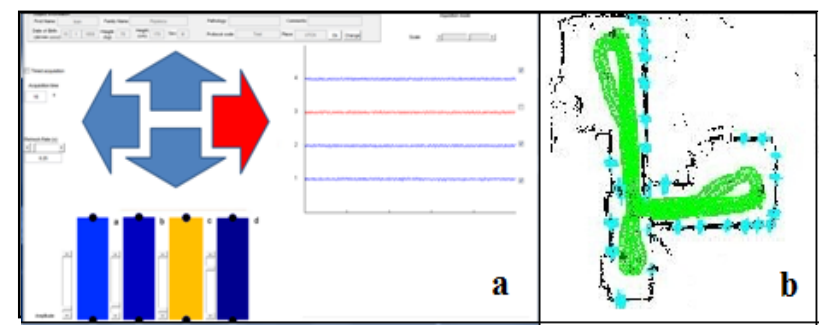

Fig. 2. (a) The grahical user interface; (b) Map obtained with the FastSLAM algorithm and the correctly identified features algorithm yielded the same result. A 0.78 succes rate (valid directions for the wheelchair per seccond) was obtained when two consecutive outputs of the neural network were considered to validate a user command.

The preliminary results of the algorithm provided important indications that, it could be used to generate valid commands to a wheelchair control system whitch would give disabled people the chance to properly use a wheelchair even in absence of their hand. Even more, the EMG sensors could be placed on any other muscles and, with proper training, allowing even the people with healty hands to make full use of them in other tasks.

The system was tested for the particle filter SLAM in three types of environments: one with sparse features, one with a medium number of features, Fig. 2. b, and a dense environment.

The algorithm produced good results for all three types of environments, the estimated landmarks were $93 \%$ in really close range of the actual landmarks positions and the average mapping time for a 100 squared meters environment was close for the three tested maps, it did not increase very much for an average number of particles used.

In conclusion, our innovative solution, by the use of EMG signals to control the wheelchiar and by the implementation of the particle filter SLAM in these conditions, gave good results in the tested environment. The EMG algorithm had a success rate of $78 \%$, while the SURF method with the particle filter correctly indentified landmarks in $93 \%$ of the cases. These results are supporting the hypothesis that this system could one day be used by humans in need of a wheelchair everywhere.

As for future developments, this solution could be applied to more extensive and complex environments by the use of a submapping algorithm for the SLAM and by the integration of a more robust path planning algorithm for the autonomous navigation of the wheelchair.

\section{ACKNOWLEDGEMENT}

This paper was supported by the project "Doctoral studies in engineering sciences for developing the knowledge based society-SIDOC" contract no. POSDRU/88/1.5/S/60078, project co-funded from European Social Fund through Sectorial Operational Program Human Resources 2007-2013.

\section{REFERENCES}

Cheein, F., Lopez N.; Soria, C.; Sciascio, F.; Pereira, F.; (2010). SLAM algorithm applied to robotics assistance for navigation in unknown environments, Journal of Neuroengineering and Rehabilitations, ISSN 1743-0003, Vol. 7, pages 503-506, United Kingdom

Jeong, H.; Zenn, B.Z.; Dae, K.; Hyong L.; Jong K.; (2003). Human-machine interface for wheelchair control with EMG and its evaluation, Proceedings of the 25th Annual International Conference of the IEEE Engineering in Medicine and Biology Society, ISBN 0-7803-7789-3, Vol. 2, pages $1602-1605$, Cancun, Mexico

Sembera, J.; Solc, F.; Vesely, M.; (2009). System of automatic loading-guiding of disabled wheelchair into a car, Annals of DAAAM \& Proceedings, 2009, ISSN: 1726-9679, Ed. Katalinic, B., pages 1091-1092, Published by DAAAM International Vienna, Vienna, Austria

Tsui, C.S.L.; Pei J., Gan, J.Q.; Huosheng H.; Kui Y.; (2007). EMG-based hands-free wheelchair control with EOG attention shift detection, Proceedings of IEEE International Conference on Robotics and Biomimetics 2007, ISBN 9781-4244-1761-2, pages 1266-1271, Sanya, China

Wang, J.; Wei, B.; Zhang, Y.; Chen, H.; (2009). Design of an autonomous mobile robot for hospital, IEEE International Symposium on IT and Medicine \& Education, ISBN: 9781-4244-3928-7, pages 1181-1186, August 2009, Jinan 